\title{
EAl Endorsed Transactions

\section{An Energy Efficient Health Monitoring System using Relay Node in Wireless Body Area Networks}

\author{
Naveen Bilandi*, Harsh K Verma, Renu Dhir \\ Department of Computer Science and Engineering, National Institute of Technology, Jalandhar, India
}

\begin{abstract}
INTRODUCTION: In today's world, Wireless Body Area Networks (WBAN) has emanated as a budding field within the healthcare scenario for monitoring, detecting, predicting, and diagnosing various fatal diseases such as cancer, cardiovascular, diabetes and other infectious diseases such as tuberclosis, pneumonia and ebola. It is an assemblage of bio-sensors which could be put on or embedded in a patient's body to surveil

the functionality of various body organs.

OBJECTIVES: The purpose of that proposed protocol is to select the relay node and record the various physiological parameters like body temperature, blood pressure, rate of blood sugar of human being. Energy consumption is a major hurdle in WBAN as replacing as well as recharging the nodes pinioned to human body may bring about discomfort to the patient. Therefore, in this work, the emphasis is given to improve the performance of WBAN i.e. network lifetime, greater mobility, stability and energy efficiency.

METHODS: To find the shortest energy-efficient route and avoid any kind of relay node failure, a novel routing scheme called Enhanced Opportunistic Protocol using Relay nodes (E-OPR) is proposed for data routing. CONCLUSION: Finally, an enhanced opportunistic protocol using relay node in WBAN has been proposed for cost effective real time health monitoring system. The proposed solution seems to give better results as compare single-hop, multi-hop routing protocols in terms of throughput, network lifetime, energy consumption and path loss.
\end{abstract}

Keywords: WBAN, E-OPR, Relay, Routing, Energy, Health-monitoring.

Received on 14 October 2019, accepted on 30 October 2019, published on 13 November 2019

Copyright (C) 2019 Naveen Bilandi et al., licensed to EAI. This is an open access article distributed under the terms of the Creative Commons Attribution license, which permits unlimited use, distribution and reproduction in any medium so long as the original work is properly cited.

doi: 10.4108/eai.13-7-2018.164098

*Corresponding author. Email:naveenb.cs.14@nitj.ac.in

\section{Introduction}

World population evolution is facing significant contests of the aging population and a rise in the expenses for health care. The accessible resources for essential services appear to be on the way out in some places. Thirty years ago, many of the people in Brazil spent on healthcare for general prevention and other healthy activities. Before ten years, when $15 \%$ of the share had fallen, then the developed countries contribute to the healthcare sector for curing chronic diseases and conventionally emphasize the healing of disease. Although many people seek care at hospitals due to the lack of personnel and supplies at health centers, almost ensuring that health centers will continue to lack the necessary resources(1-6). But the problem of traveling along with expenses led to the rise in the number of deaths of the patients. The poor elderly people in various countries are unable to manage the rising expenses of health care, which is the vital point to start beginning new technology to existing health care applications. Time-sensitive illnesses and injuries cause a significant burden of diseases in 
developing countries. In developing countries, there is no provision of priority for timely treatment during life-threatening emergencies. An effective level of emergency medical care improves the health of populations along with actual community needs. At present, all these needs are achieved by the great invention in the medical field by the use of wireless sensor networking. Networking enabled communication media for both the patient and the doctor by the use of sensor networks. Sensor networks aimed to provide a mode for the exchange of information by sensing the variation in the body with utmost efficiency. parameters of the patient and hence achieving a level of comfort $(7,8)$. The technique behind the comfort level is hidden to the patient as a response is very much faster (in $\mathrm{ms}$ ), which is achieved by the functions performed by the actuators. All these health-related-work is done by the WBAN invented by Van Dam in 2001.The potential field of Body Area Networks gives new meaning to the term "delicate" in the sense of health monitoring. The health of an individual is very crucial and needs timely and constant monitoring. WBAN adhere to a category called external monitoring health care systems (eHealth), which are used to perform local monitoring and controlling. A WBAN system offers long term health monitoring without hindering day-to-day activities. WBANs are concurrently operating, less-energy, low-ranged network. It comprises various types of intelligent, low-power, contracted biosensors which may be attached over the clothes or on the body or may be implanted inside the skin. They possess the capability of accumulating physiological data, namely electroencephalography (EEG), electrocardiography (ECG), arterial pressure, blood sugar, temperature, etc.

\subsection{Applications of WBAN}

\section{Electroencephalography (EEG):}

Electroencephalography represents the electrical patterns of the human brain's activity. Recently, the ambulatory-EEG (A-EEG) patterns have supported significantly in the diagnosis of diseases like narcolepsy, epilepsy, etc. and also to monitor the responses of treatments as well. EEG sensors record and analyze the A-EEG signals during daily chores, thereby being less botheration for the patient.

\section{Electromyography (EMG):}

Electromyography monitors and analyzes the signals emitted by the body muscles. The signals are captured by an electrode during muscle contraction. During muscle contraction, electric signals are paved. The aggregation of these signals constitutes the EMG signals. An EMG sensor is used to diligently monitor muscle activity.

Diabetes: Diabetes is a disease that occurs when there are alarmingly high blood-sugar levels. It can be type-I diabetes or type-II diabetes. The diabetes sensor effectively indicates any fluctuation in the blood glucose or blood sugar levels and keeps a close check on the patient's levels.

Cancer Detection: Cancer patients are projected to rise by more than $50 \%$ by 2025 . WBAN sensors can thus be capable of monitoring and early detection of any tumors before they become malignant. This can save the patient from the rigorous chemotherapy sessions.

\section{Electrocardiography (ECG):}

An electrocardiogram generates a wave-form which monitors the electrical activity of the heart. The ECG sensors can effectively monitor heartbeat of the patient even on the go. This can significantly benefit patients at any time.

\section{Blood Pressure:}

The Blood Pressure comprises of two pressures, namely systolic and diastolic pressures. The BP sensor monitors the blood pressure levels and indicates any abrupt rise or fall in a patient's BP.

\subsection{WBAN Components for Health Monitoring}

This network is the subnet of wireless sensor networks. WBAN aimed to provide flawless communication between a patient and the doctor wirelessly without being physically present at the hospital. The patient's mobility doesn't affect his monitoring. WBAN, as a part of WSN, consists of numerous tiny sensors, Actuators, and PDA. All these works together for transmitting the data to remote places like hospitals. The key components of WBAN are:

Sensors: The principal purposes of the sensor nodes are to unremarkably section vital signs and send the relevant data to a medical server using IEEE 802.15 .1

Personal Device Assistance (PDA): The PDA holds the personal medical server which controls the WBAN along with providing the GUI to the patient and hence transfers the sensed data to the personal server through the use of GPRS and internet 
Actuators: The actuator interrelates with the patient upon unloading statistics from the sensor node(9).

The whole record (an electrical record) of the registered users is kept at the medical server, which helps the users by providing ease of access. Also, the records are safely stored, and only the particular user can access his/her records - deployment of sensor nodes in the human body shown as figure 1 .

\subsection{Challenges in WBAN}

There are numerous challenges in WBAN, but the most important are:

Quality of service (QoS): The WBAN must ensure the Quality of Service to its users in the form of Reliability, Data Availability, Fault Tolerance, energy Consumption, Synchronization, etc.(10-14).

Routing: The data from one sensor node to another or to the sink must be adequately routed without any delay. In case of failure in between the data transmission, the network must be self-configurable for adopting any changes (15).

Security: It is the primary concern as data must be stored securely so that no one can access the user's data without his/her permission. data-authentication, data-integrity, data-confidentiality, and datafreshness must be guaranteed for the user.

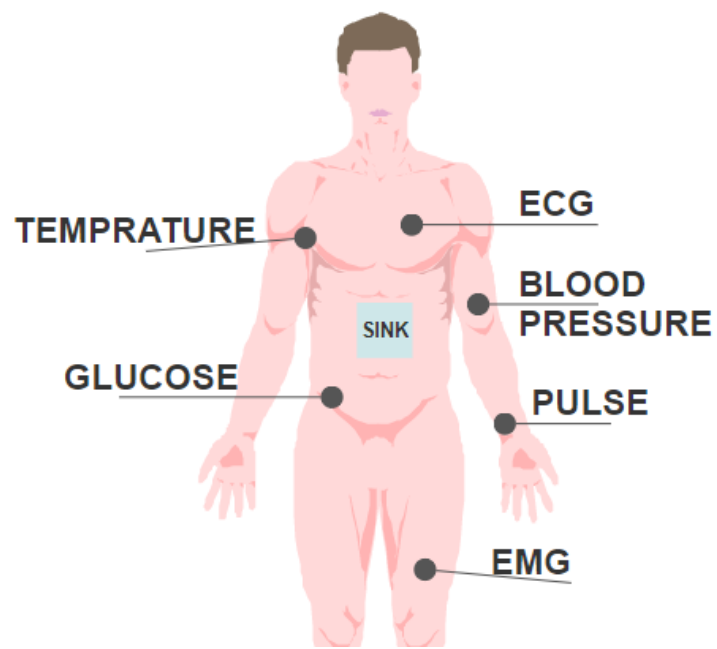

Figure 1. Sensor placement at human body

\subsection{Novelty and contribution of proposed research work}

The main challenges are the energy of network that is based on routing challenges like movements of the body (postural movement), limitation of resources, temperature- rise and interfering, heterogeneous environment, and dynamic transmission range(16). For this problem, we proposed a novel and energyefficient routing protocol to select the relay node(17-21). For WBAN, an opportunistic protocol following the dynamic adjustment of relay nodes using predefined relaying nodes is presented here(18). This protocol is designed for the applications which need the periodic transmission of data from sensors to the sink; also, it supports star network topology. In this, a dedicated time slot is assigned to each sensor node for direct transmission to the sink in the standard period. When the direct transmission failed, the relay node is selected dynamically for successful data transmission. The nodes which failed in the direct transmission process during a reasonable period follow the relay period in which the relay process is executed. The node which occupies the initial slot has the more chance to find a relay node, while a node which occupies the last time slot has no possible relaying node. Also, here predefined relay nodes are introduced, which are work alternatively if any of the selected relay nodes do not perform the data relay process. This protocol improves the probability of successful data relaying and also improves the network lifetime as compare to existing routing protocols such as SIMPLE (22), M-ATTEMPT (23), Relay based protocol (24).

The numerical outcomes depict that model in the following way. In Section2, related work is presented. Section 3 describes the WBAN design model. The details of the proposed protocol are presented in section 4. Section 5, shows the simulation results and section 6 , conclude the paper.

\section{Related Work}

In WBAN, numerous routing mechanisms were proposed for minimizing of energy consumption and improving network life time using selection of relay node. Chavva et al. (25) proposed an energy efficient routing protocol in wireless body area network by reducing the energy consumption using fuzzy approach. It uses multi-hop routing technique for sensing the data and send to the sink. Huang et al. (26) proposed a technique is for selecting an appropriate path to enhance remnant energy-levels for WBAN. Bhardwaj et al. (27) proposed the health monitoring framework closer to WBAN users which is the amalgam of autonomic-computing and queuing-model. K. Shashi Prabh et al.(28) presented 
a collision-free body area network MAC protocol it monitors and predicts the fluctuations in the channel. This protocol is capable of resolving co-channel interference when multiple co-located BANs got introduced in the nearby region. It is noted that BANMAC is flexible. Hui Feng et al.(29)proposed a prediction-based dynamic relay transmission (PDRT) scheme. In PDRT, a fixed slot assignment was done rather than a variable slot assignment for enhancing the performance. PDRT improves energy efficiency and transmission reliability. In terms of Ehealth care system, Romero et al. (30) proposed the human data interactions and human computer interaction for sociotechnical health monitoring system. Jagadeeswari et al. (31) analysed that upcoming techniques for medical healthcare system to improve the quality of life. It provide E-heath services by focusing on internet of things, cloud computing, bigdata analytics and applications which is based on WBAN. Nadeem et al.(22) presented a Stable Increased-Throughput Multi-Hop protocol for Link Efficiency (SIMPLE), which used multihop topology by deploying eight sensor nodes on the human body. They describe a cost function for evaluating the parent node and the forwarder node. The parent node was selected based on minimum distance from the sensor node to sink and maximum residual energy. In the initial phase, the sink broadcasts packet containing its location to other nodes so that all can store its information. In the node deployment phase, each sensor nodes broadcast packet containing their information so that their neighbors can store their information. SIMPLE maximize the network stability period and high packet delivery. N. Javaid et al. (23) presented a routing protocol named Mobility-supporting Adaptive Threshold-based Thermal-aware Energyefficient Multi-hop Protocol. It routes the data gone from the link Hot-spot. In the initial phase, all nodes update their neighbors, sink position, and available routes to the sink node. In the routing phase, the best route was selected on the basis of energy consumption. The nodes with a high data rate send data directly to the sink node. It allows minimum energy consumption and better reliability for packet delivery. Marshed Mohamed et al. (32) analyzed the dynamic features of wireless body area network channel while walking, while balancing for complex factors and movement of body. Javaid et al.(24) a protocol based upon the relay mechanism for sensors node placed in the patient body. Authors adopted a mathematical linear programming model for maximizing the network lifetime and minimizing the end-to-end delay. The communication distance between in-body sensors was decreased to their minimum value. Also, no multi-hoping was allowed among in-body sensors. They placed relays on the body of the patient for easy recharging in case of battery degradation and easy replacement in case of relay failure. In (33) presents a novel approach for reducing the consumption of energy in wireless body area network. This approach is used for balancing the energy of consecutive sensing and transmitting signal toward the sink. In order to diagnosis, Sharma et al. (34) presented the opportunities and challenges for diagnosis of psychiatric disorder using soft computing algorithm. From the literature survey, it has been concluded that there are numerous protocols in WBAN for health monitoring purposes. All the protocols hold the major issue of routing and energy, as it is the primary concern in WBAN, which is being resolved in this paper with the concept of relay nodes.

\section{Design Model for Wireless Body Area Networks}

Before explaining the methodology, it is important to know the various phases of the design model of WBAN.

\subsection{Network Models}

WBAN holds Multi-tier communication architecture. The model of the network can be differentiated in three tiers shown in figure 2.

a) Setup Phase (Layer-1): Intra-WBAN Network.

b) Execution Phase (Layer-2): Inter-WBAN Network.

c) Analysis Phase (Layer-3): Beyond-WBAN Network. 


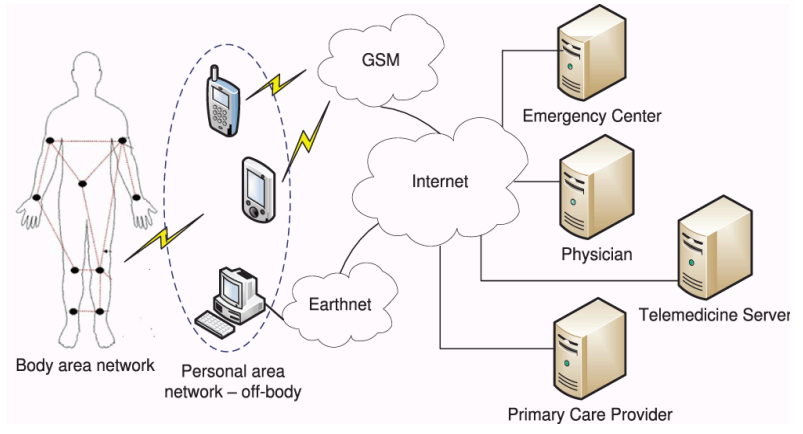

Fig. 2. WBAN design model for healthcare

In WBAN, these below mentioned phases are required for packet forwarding.

Setup Phase: The network is deployed with the sensor nodes. In the deployed network sink node is created to gather the sensed information.

Execution Phase: In the network, some relay nodes are deployed, which sense the body information and passes that information to a base station. In the network, the fault may occur on the sensor nodes. This technique selects the best relay node on the basis of energy and resources for fault recovery.

Analysis Phase: The simulation is performed, and it has been analysing that the proposed algorithm performed very well in various parameters like energy(35-38), network lifetime, PDR, routing overhead, and packet loss.

During the movement of the human body, if any child node moves away from its parent node, then it gets associated with another nearest parent node to save energy. Hot-spot condition occurs when excessive heat is sensed from the nodes.

\subsection{Energy Model for WBAN}

In the energy model, the consumption of energy is minimized by analyzing it. In WBAN, a node is of two types: Forwarder node and Normal node. As multiple tasks are performed by the forwarder node, hence its energy consumption varies according to the tasks(39).

The total amount of transmit energy of relay node (forwarder node) is calculated by given Eq. (1)

$E_{f w d}^{T x}=N \emptyset E_{T x}(l, d)+N l \emptyset E_{\text {elec }}$

Where,

$\emptyset$ : Data aggregation factor

$l$ : Packet size,
$N$ : Number of sensor nodes

$E_{T x}$ : Transmitter Energy consumption

$E_{\text {elec }}$ : Energy consumption by electronics circuit.

The total amount of transmit energy of relay node utilized for the entire lifetime is evaluated by Eq. (2)

$E_{n t w}^{T x}=\int_{0}^{t} E_{f w d}^{T x} d t$

Total transmit energy of sensor node evaluated by Eq. (3)

$E_{\text {nor }}^{T x}=E_{R x}(l)$

where,

$E_{R x}$ : Receiver energy consumption by the receiver for each packet.

In the same way, the total amount of transmit energy of every sensor node utilized as given below Eq. (4)

$E_{\text {ntw }}^{T x}=\int_{0}^{t} \sum_{n-1} E_{\text {nor }}^{T x} d t$

The total amount of reception energy of relay node calculated by given Eq. (5), in which the amount of data received by a forwarder node and the number of nodes is expressed as:

$E_{f w d}^{R x}=(N-1) E_{R x}(l)$

The total amount of reception energy of relay node utilized for the entire lifetime is evaluated by Eq. (6) $E_{f w d \_n t w}^{R x}=\int_{0}^{t} E_{f w d}^{R x} d t$

The nodes having maximum distance from the sink always lead to higher consumption of energy. Therefore, the node at a maximum distance gets a chance of becoming a forwarder node.

\section{Proposed Approach}

The wireless sensor network is the kind of network that is used to sensing the environmental conditions like temperature, pressure, etc. The WBAN is the network that is used for healthcare diagnose. After sensing the condition of the human body, the retrieved information is passed to the transmitter. The transmitter, after receiving the sensed information, performs required actions according to human body conditions. The trouble is found in the wireless body area network is FAULT. The fault may occur due to the failure of sensors and due to battery degradation of the sensors. To recover fault, a scheme of relay nodes as an intermediate is adopted along with the sensor nodes. When the sensor node gets dead, or battery of the sensor node got degraded, relay nodes are responsible for redirecting the sensor node traffic. The main problem that occurred in this technique is of complexity. As the network has a limited number of sensors, fault condition leads to the network delay 
and routing overhead. In this work, further enhancement in the relay-based technique for fault recovery in the network is proposed.

\subsection{Proposed Algorithm}

The proposed algorithm for the E-OPR protocol for energy-efficient is shown in Algorithm 1.

\subsection{Methodology}

The wireless body area network is a kind of network that is used to sense body conditions. The sensors are deployed in such a manner that they can efficiently sense parameters of the body and then passed the data to the sink. Communication in the wireless body area network is multi-hop communication. In this type of communication, the source node establishes the shortest path to the destination. For that purpose, the source node can use some non-critical sensor nodes for transmitting their data further to the sink. These non-critical sensor nodes are those who sense some non-critical parameters like temperature, pressure, altitude, etc. Mostly they remain in the sleep mode; that's why they are forced to participate in the communication purpose. But in case these nodes get faulty, or battery of these nodes gets degraded, Fault may arise in the network. The relay-based technique uses the relay nodes for fault recovery. For the purpose of achieving better results, when link is failure during communication a Destination Sequenced Distance Vector (DSDV) and Enhancement in Ad-hoc Ondemand Multipath Distance Vector (EAOMDV) (40) routing protocol is put forth to optimize a routing path for E-health application.

The proposed improvement is based on dynamic routing. In this routing, multiple paths are established from the source to the destination.

When the battery of any sensor node reduced to the threshold value, a new path will be automatically chosen for data transmission. Nodes transfer data through an intermediate node to the sink. The existing technique leads to a reduction in the network complexity, network delay, energy consumption, and hence increased the network throughput. Due to the fault, network performance degrades in terms of throughput, packet loss, delay, and energy. In the proposed technique, the enhancement is done by considering the predefined relay nodes. Three scenarios are depicted to prove the variation in the results by considering a different number of nodes each time.

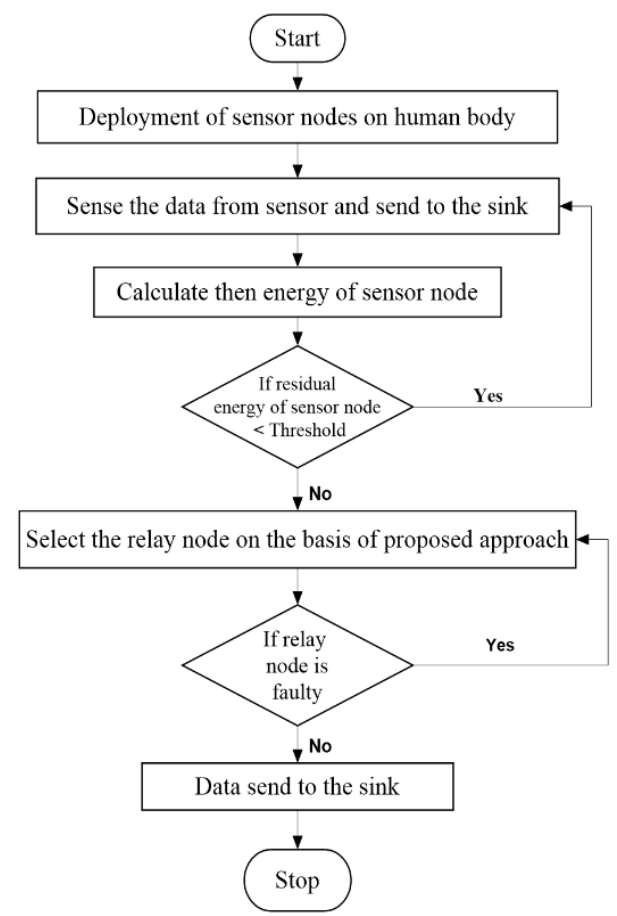

Fig. 3. Proposed approach of WBAN

\subsection{Simulation Scenarios of Proposed Approach}

In this simulation process, two scenarios have been evaluated on the basis of different parameters. In this methodology, 2 scenarios are implemented in the simulation tool. In simulation scenarios having sensor 1 is equipped with the functionality of measuring eating and smoking habits called as Tooth Sensor. Sensor 2 is the ECG Sensor here used for monitoring heart activity. Sensor 3 is the Health Patch that is placed at the chest and measures the parameters like heart-rate, heart-rate variability, temperature, body-posture, steps, and falls. Sensor 4 is the Limb sensor that monitors the movements of the legs of the human. Sensor 5 is equipped with the capability of tracking the wearer's steps, speed, foot landing technique, weight distribution, and altitude and known as the Smart Sock. Here, Sensor 6 is the Sink node capable of accepting packets from all other sensor nodes and hence forwards them to the base station. Also, it accepts the computed information from the base station and helps in responding to critical conditions. All this response is in the form of an action that will be done by the 
actuator, which will inject medicine in the human body if required.

Algorithm 1. Proposed Algorithm for E-OPR

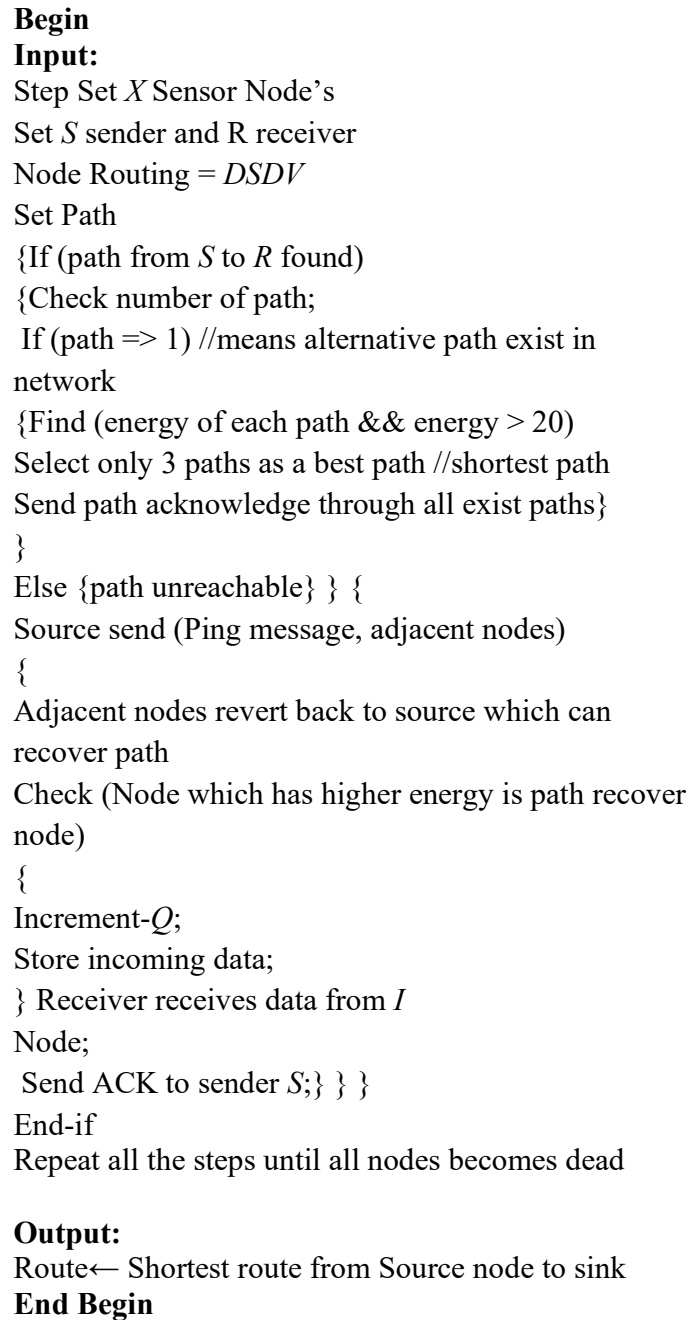

Scenario 1: No extra node is deployed, which acts as a relay node. All other sensor nodes are the same as that of the previous scenario. In this, six sensor nodes, along with one sink, is present.

Scenario 2: Here, nodes are present along with predefined relay nodes and a single sink, which helps in forwarding packets to the sink by accepting from the relayed nodes if any of the intermediate nodes (sensor node used as a relay node if no such node is present in the network and in case if it is not sensing at that time) gets faulty or corrupted. These are helpful in emergency cases. These nodes are not the sensor nodes but just used for the relaying process. Throughput gives the best performance in this scenario. Variables of Simulation is given below in Table 1.
Table 1. Variables of Simulation for WBAN

\begin{tabular}{|c|c|}
\hline Parameter & Values \\
\hline Nodes & 6 \\
\hline Simulation Area & $800 * 800$ \\
\hline Simulation-Time & 8000 rounds \\
\hline Type of Antenna & Omni-Directional \\
\hline MAC- Layer & $802.11 \mathrm{e}$ \\
\hline Type of Channel & Wireless Channel \\
\hline Routing -Protocol & DSDV \\
\hline Physical -Medium & Wireless \\
\hline
\end{tabular}

\section{Results Analysis}

The execution of the proposed protocol is evaluated here, after analyzing the performance of the residual energy without relay by using numerous metrics like throughput, energy, network lifetime. Initially, the energy of all nodes is kept constant ( $1 \mathrm{~J}$ per node)

\subsection{Throughput}

Throughput is computed as the size of bits (total bits) divided into the simulation duration (duration). As illustrated in Fig. 4, the throughput of the opportunistic protocol is high as compare to the other scenario. By considering the relay nodes, throughput increased. As the relay nodes help in maintaining the network uniformity by allowing the packets to reach the sink, the packet drop ratio gets reduced.

Simulation duration $=$ time End - time Begin

where, time Begin = time of the first line in the trace file time End= time of the last line in the trace file

\subsection{Energy Consumption}

It is the total consumed energy of each sensor node placed in the body(41). Fig. 4 shows the energy comparison between without relay mechanism and selection of relay mechanism of proposed schemes. 
This kind of protocol ensures maximum energy in the nodes that resulting in the transmission of data from transmitter to receiver. It also increased the productivity of the network. In scenario 1 , due fault in the network, packet-loss is increased at a steady rate.

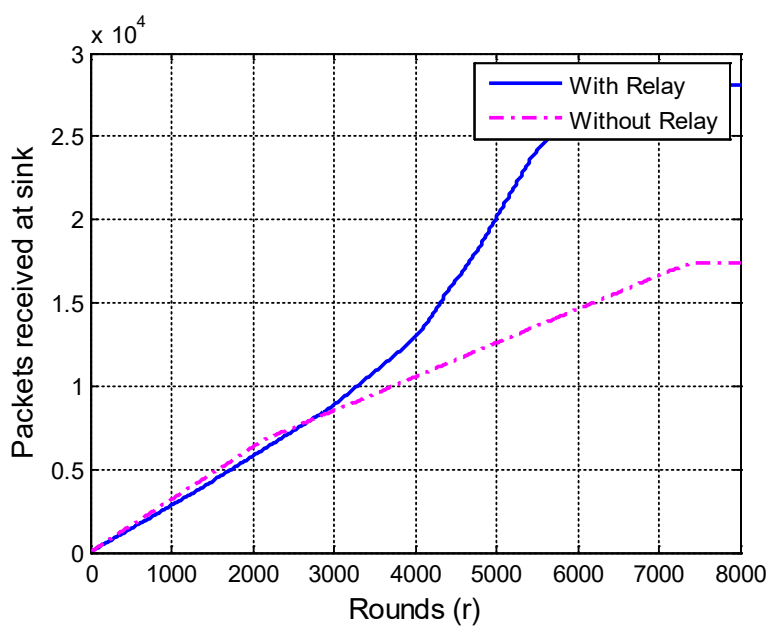

Fig. 4. Analysis of throughput with respect to rounds

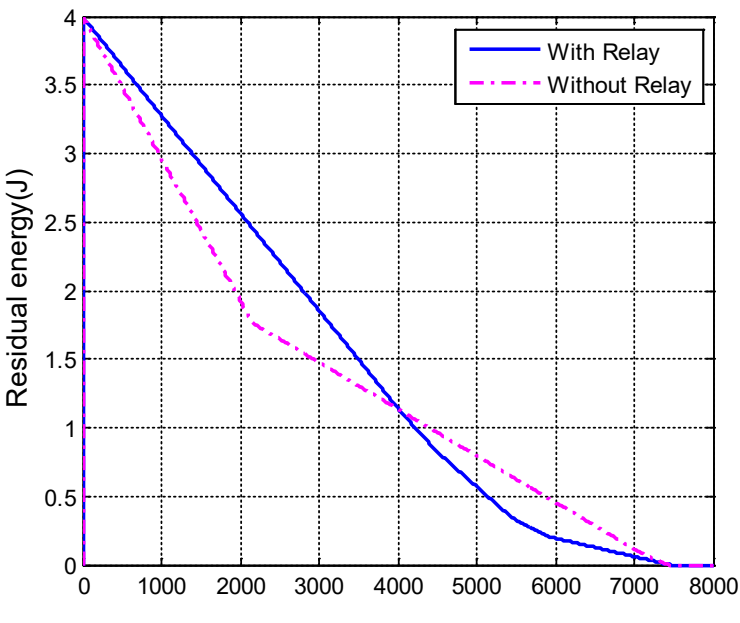

Fig. 5. Analysis of energy with respect to rounds

Hence, packing forwarding again and again due to the fault leads to more energy consumption. The proposed technique recovered the network fault and finally led to a reduction in the energy consumption of the network. Figure 5 shows minimum energy consumption.

\subsection{Network Lifetime}

The network stability period is the duration of time in which network operations are performed until the nodes die (42). As far as the medial applications are concerned, the network should be optimized for lifetime. Most of the wireless sensor networks utilize various energy-efficient protocols for communication among the nodes and base stations. The proposed protocol has more stability period. It plays vital role for balancing the energy consumption and increase the network lifetime. The network should be operated for as long as it is required for measuring the body parameters.

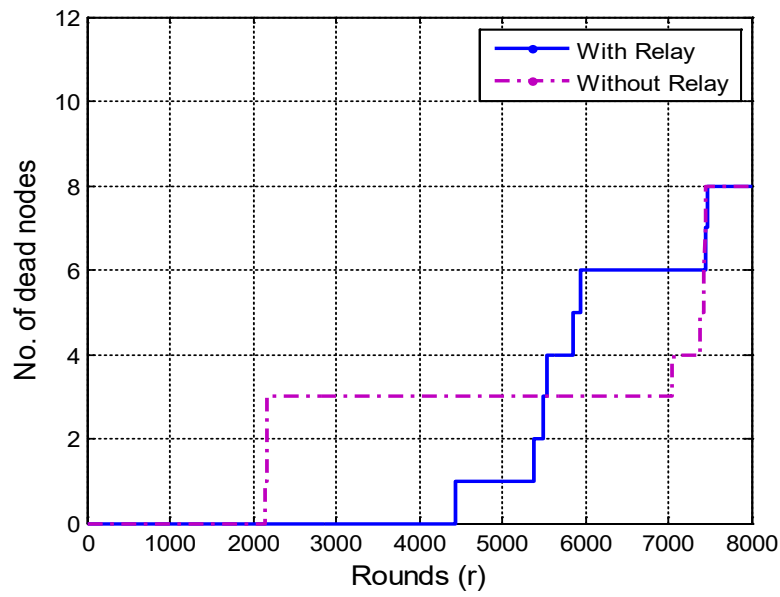

Fig. 6. Analysis of network lifetime with respect to rounds

Hence, Energy degradation leads to a halt in the network operational time (43).In Fig. 6, the network lifetime is increased due to fault recovery in the network by using relay nodes.

\subsection{Path loss}

Path loss is defined as the reduction in power for propagation while transmission of the data from sensor node to the sink. During propagation, sometimes the signals are diverted from the destination and split in the no. of fragmentation. The diversion of the signal is known as Path loss. Basically, it is loss of power density. This term is mostly used in the wireless network for transmission of data over the network. Basically, it is loss of power density.

The overall results comparison of scenario 1 and scenario 2 with different parameters is given in Table 2 . 


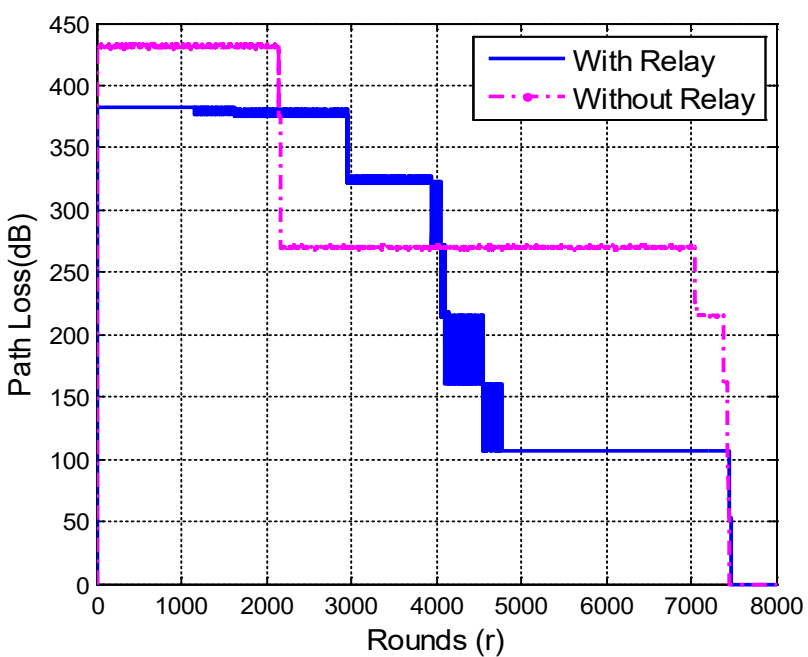

Fig. 7. Analysis of path loss with respect to rounds

Table 2. Result analysis of scenario 1 and scenario 2 with different parameters

\begin{tabular}{|c|c|c|c|}
\hline \multicolumn{2}{|c|}{ Parameters } & $\begin{array}{l}\text { Scenario: } 1 \\
\text { (Without } \\
\text { Relay) }\end{array}$ & $\begin{array}{l}\text { Scenario: } 2 \\
\text { (With } \\
\text { Relay) }\end{array}$ \\
\hline \multicolumn{2}{|c|}{$\begin{array}{l}\text { No of rounds at which first } \\
\text { sensor node dies }\end{array}$} & $\begin{array}{l}215 \\
0\end{array}$ & $\begin{array}{l}437 \\
0\end{array}$ \\
\hline \multicolumn{2}{|c|}{$\begin{array}{l}\text { No of rounds at which last } \\
\text { sensor node dies }\end{array}$} & $\begin{array}{l}723 \\
5\end{array}$ & $\begin{array}{l}725 \\
5\end{array}$ \\
\hline \multicolumn{2}{|c|}{ Packets received } & $\begin{array}{l}175 \\
52\end{array}$ & $\begin{array}{l}280 \\
11\end{array}$ \\
\hline \multirow{8}{*}{$\begin{array}{l}\text { Res } \\
\text { idu } \\
\text { al } \\
\text { Ene } \\
\text { rgy } \\
(\mathbf{J})\end{array}$} & $\begin{array}{l}\text { At } 1000 \\
\text { rounds }\end{array}$ & 3.01 & 3.30 \\
\hline & $\begin{array}{l}\text { At } 2000 \\
\text { rounds }\end{array}$ & 1.90 & 2.60 \\
\hline & $\begin{array}{l}\text { At } 3000 \\
\text { rounds }\end{array}$ & 1.50 & 1.90 \\
\hline & $\begin{array}{l}\text { At } 4000 \\
\text { rounds }\end{array}$ & 1.21 & 1.24 \\
\hline & $\begin{array}{l}\text { At } 5000 \\
\text { rounds }\end{array}$ & 0.73 & 0.80 \\
\hline & $\begin{array}{l}\text { At } 6000 \\
\text { rounds }\end{array}$ & 0.51 & 0.52 \\
\hline & $\begin{array}{l}\text { At } 7000 \\
\text { rounds }\end{array}$ & 0.23 & 0.24 \\
\hline & $\begin{array}{l}\text { At } 8000 \\
\text { rounds }\end{array}$ & 0 & 0 \\
\hline \multirow{4}{*}{$\begin{array}{l}\text { Path- } \\
\text { Loss (dB) }\end{array}$} & $\begin{array}{l}\text { At } 2000 \\
\text { rounds }\end{array}$ & $\begin{array}{l}43 \\
7\end{array}$ & $\begin{array}{l}38 \\
1\end{array}$ \\
\hline & $\begin{array}{l}\text { At } 4000 \\
\text { rounds }\end{array}$ & $\begin{array}{l}27 \\
5 \\
\end{array}$ & $\begin{array}{l}27 \\
5\end{array}$ \\
\hline & $\begin{array}{l}\text { At } 6000 \\
\text { rounds }\end{array}$ & $\begin{array}{l}27 \\
5\end{array}$ & $\begin{array}{l}11 \\
0\end{array}$ \\
\hline & $\begin{array}{l}\text { At } 8000 \\
\text { rounds }\end{array}$ & 0 & 0 \\
\hline
\end{tabular}

\section{Conclusion and Future work}

In WBAN, there are numerous sensors and relay nodes for monitoring body parameters and data transmission, respectively. The relay nodes play a significant role as an inter-mediator. Due to the wrong selection of the relay node, there are always chances of the failure in the transmission of data from the sensor node toward the sink. For that purpose, relay nodes are there for packet forwarding toward the sink. The relay-nodes are selected from the nearest neighborhood along with considering maximum energy. An attempt is made on the selection of the relay node so that the failure may not occur, and hence data will be transferred to the sink without delay. The proposed technique is based on energy and resources in terms of buffer size. In future proposed technique will be applied hybrid MAC protocols for channel sensing and further enhancements can be done for the dynamic adjustments of the sensor nodes in WBAN and it can be used in the field of diagnosis of kidney dialysis, diabetes and other general prevention.

\section{References}

[1] Effatparvar M, Dehghan M, Rahmani AM. A comprehensive survey of energy-aware routing protocols in wireless body area sensor networks. J Med Syst. 2016;40(9):201.

[2] Demir SM, Al-Turjman F, Muhtaroglu A. Energy Scavenging Methods for WBAN Applications: A Review. IEEE Sens J. 2018;18(16):6477-88.

[3] Khan NA, Javaid N, Khan ZA, Jaffar M, Rafiq U, Bibi A. Ubiquitous HealthCare in wireless body area networks. Proc 11th IEEE Int Conf Trust Secur Priv Comput Commun Trust - 11th IEEE Int Conf Ubiquitous Comput Commun IUCC-2012. 2012;1960-7.

[4] Latré B, Braem B, Moerman I, Blondia C, Demeester P. A survey on wireless body area networks. Wirel Networks. 2011;17(1):1-18.

[5] Maity S, Das D, Sen S. Wearable Health Monitoring Using Capacitive Voltage-Mode Human Body Communication. 2017;2-5.

[6] Yuce MR. Implementation of wireless body area networks for healthcare systems. Sensors Actuators, A Phys. 2010;162(1):116-29.

[7] V. Crosby G. Wireless Body Area Networks for Healthcare: A Survey. Int J Ad hoc, Sens Ubiquitous Comput. 2012;3(3):1-26.

[8] Maman M, Denis B, D'Errico R. Research trends in wireless body area networks: From On-Body to Body-to-Body cooperation. In: International Symposium on Medical Information and Communication Technology, ISMICT. Italy: IEEE; 2014;1-5.

[9] D'Andreagiovanni F, Nardin A. Towards the fast and robust optimal design of wireless body area networks. Appl Soft Comput J. 2015;37:971-82.

[10] Moosavi H, Bui FM. Optimal relay selection and power control with quality-of-service provisioning in wireless body area networks. IEEE Trans Wirel Commun. 2016;15(8):5497-510. 
[11] Meshkati F, Poor HV, Schwartz SC, Balan R V. Energy-efficient resource allocation in wireless networks with quality-of-service constraints. IEEE Trans Commun. 2009;57(11):3406-14.

[12] Otal B, Verikoukis C, Alonso L. Highly Reliable Energy-saving MAC for Wireless Body Sensor Networks in Healthcare Systems. IEEE J Sel Areas Commun Spec Issue Wirel Pervasive Commun Healthc. 4293;27(4):553-65.

[13] Samanta A, Misra S. Dynamic Connectivity Establishment and Cooperative Scheduling for QoSAware Wireless Body Area Networks. IEEE Trans Mob Comput. 2018;17(12):2775-88.

[14] Raja KS, Kiruthika U. An Energy Efficient Method for Secure and Reliable Data Transmission in Wireless Body Area Networks Using RelAODV. Wirel Pers Commun. 2015;83(4):2975-97.

[15] Yuan X, Li C, Yang L, Yue W, Zhang B, Ullah S. A token-based dynamic scheduled MAC protocol for health monitoring. EURASIP J Wirel Commun Netw [Internet]. 2016;2016(1):125.

[16] Movassaghi S, Abolhasan M, Lipman J, Smith D, Jamalipour A. Wireless Body Area Networks: A Survey. Ieee Commun Surv Tutorials. 2014;16(3):1658-86.

[17] Zhang Y, Zhang B. A Relay-Aided Transmission Power Control Method in Wireless Body Area Networks. IEEE Access. 2017;5:8408-18.

[18] Pan R, Chua D, Pathmasuntharam JS, Xu YP. An Opportunistic Relay Protocol with Dynamic Scheduling in Wireless Body Area Sensor Network. IEEE Sens J. 2015;15(7):3743-50.

[19] Vasudevan S, Adler M, Goeckel D, Towsley D. Efficient algorithms for neighbor discovery in wireless networks. IEEE/ACM Trans Netw. 2013;21(1):69-83.

[20] Sui D, Hu F, Zhou W, Shao M, Chen M. Relay Selection for Radio Frequency Energy-Harvesting Wireless Body Area Network With Buffer. IEEE Internet Things J. 2018;5(2):1100-7.

[21] Roopali RK. Energy Efficient Dynamic Cluster Head and Routing Path Selection Strategy for WBANs. Wirel Pers Commun. 2020;1-26.

[22] Nadeem Q, Javaid N, Mohammad SN, Khan MY, Sarfraz S, Gull M. SIMPLE: Stable increasedthroughput multi-hop protocol for link efficiency in Wireless Body Area Networks. In: Proceedings 2013 8th International Conference on Broadband, Wireless Computing, Communication and Applications, BWCCA 2013. France: IEEE; 2013.p. 221-6.

[23] Javaid N, Abbas Z, Fareed MS, Khan ZA, Alrajeh N. M-ATTEMPT: A New Energy-Efficient Routing Protocol for Wireless Body Area Sensor Networks. Procedia Comput Sci. 2013;19:224-31.

[24] Javaid N, Ahmad A, Khan Y, Ali Z, Turki K, Alghamdi A. A Relay Based Routing Protocol for Wireless In-Body Sensor Networks. Wirel Pers Commun. 2015;80:1063-1078.

[25] Reddy S, Ravi C, Sangam S. An energy - efficient multi - hop routing protocol for health monitoring in wireless body area networks. Netw Model Anal Heal Informatics Bioinforma. 2019;9(1):21.

[26] Huang X, Wu Y, Ke F, Liu K, Ding Y. An energyefficient and reliable scheduling strategy for dynamic WBANs with channel periodicity exploitation. IEEE Sens J. 2019;20(5):2812-24.
[27] Bhardwaj T, Sharma SC. Sustainable Computing: Informatics and Systems Cloud-WBAN: An experimental framework for Cloud-enabled Wireless Body Area Network with efficient virtual resource utilization. Sustain Comput Informatics Syst. 2018;20:14-33.

[28] Prabh KS, Royo F, Tennina S, Olivares T. BANMAC: An Opportunistic MAC Protocol for Reliable Communications in Body Area Networks. In: 2012 IEEE 8th International Conference on Distributed Computing in Sensor Systems. China: IEEE; 2012. p. 166-75.

[29] Feng H, Liu B, Yan Z, Zhang C, Chen CW. Prediction-based Dynamic Relay Transmission Scheme for Wireless Body Area Networks. In: 2013 IEEE 24th Annual International Symposium on Personal, Indoor, and Mobile Radio Communications (PIMRC). London; 2013. p. 253944.

[30] Romero N, Sharma M. EAI Endorsed Transactions Designing human data interactions in Healthcare. 2018;4(16):2-4.

[31] Jagadeeswari V, Subramaniyaswamy V, Logesh R, Vijayakumar V. A study on medical Internet of Things and Big Data in personalized healthcare system. Heal Inf Sci Syst. 2018;6(1):14.

[32] Mohamed M. Characterization of dynamic wireless body area network channels during walking. EURASIP J Wirel Commun Netw. 2019;104(1-12).

[33] Bilandi N, Verma HK, Dhir R. PSOBAN : a novel particle swarm optimization based protocol for wireless body area networks. SN Appl Sci. 2019;1(11):1492.

[34] Sharma M, Romero N. EAI Endorsed Transactions Future Prospective of Soft Computing Techniques in Psychiatric Disorder Diagnosis. 2018;4(15):2-4.

[35] Yi C, Wang L, Li Y. Energy Efficient Transmission Approach for WBAN Based on Threshold Distance. IEEE Sens J. 2015;15(9):5133-41.

[36] Smail O, Kerrar A, Zetili Y, Cousin B. ESR : Energy aware and Stable Routing protocol for WBAN networks To cite this version: ESR : Energy aware and Stable Routing protocol for WBAN networks. In: 2016 International Wireless Communications and Mobile Computing Conference (IWCMC). Cyprus: IEEE; 2016. p. 452-7.

[37] Ullah Z, Ahmed I, Khan FA, Asif M, Nawaz M, Ali $\mathrm{T}$, et al. Energy-efficient harvested-aware clustering and cooperative routing protocol for WBAN (EHARP). IEEE Access. 2019;7:100036-50.

[38] Qais MH, Hasanien HM, Alghuwainem S, Nouh AS. Coyote optimization algorithm for parameters extraction of three- diode photovoltaic models of photovoltaic modules. Energy. 2019;187:116001.

[39] Ahmed S, Javaid N, Yousaf S, Ahmad A, Sandhu MM, Imran M, et al. Co-LAEEBA: Cooperative link aware and energy efficient protocol for wireless body area networks. Comput Human Behav. 2015;51:1205-15.

[40] Sharma I, Pundir S. Enhancement in AOMDV Protocol to Reduce Chances of Link Failure in Mobile Adhoc Network. 2016;6(2):17-20.

[41] Kaur N, Singh S. Optimized cost effective and energy efficient routing protocol for wireless body area networks. Ad Hoc Networks. 2017;61:65-84.

[42] Gondogdu K, Calhan A. An Implementation of Wireless Body Area Networks for Improving 
Priority Data Transmission Delay. J Med Syst. 2016;40(3):75.

[43] Franco F Di, Tinnirello I, Ge Y. 1 Hop or 2 Hops : Topology Analysis in Body Area Network. In: 2014 European Conference on Networks and Communications (EuCNC). Italy: IEEE; 2014. p. 15. 